\title{
Hybrid Enterprise Modelling: Integrating Modelling Mechanisms for Socio-Technical Systems Analysis and Design
}

\author{
Amjad Fayoumi ${ }^{1}$, Pericles Loucopoulos ${ }^{2}$, Ayham Fayyoumi ${ }^{3}$ \\ ${ }^{1}$ School of Business and Economics, Loughborough University, Loughborough, UK; ${ }^{2}$ Department of Informatics and Telematics, \\ Harokopio University of Athens, Athens, Greece; ${ }^{3}$ Department of Information Systems, Al-Imam Muhammad ibn Saud Islamic Uni- \\ versity, Riyadh, KSA. \\ Email: a.fayoumi@lboro.ac.uk, p.loucopoulos@hua.gr, a.fayyoumi@ccis.imamu.edu.sa
}

Received November $21^{\text {st }}$, 2013; revised December $17^{\text {th }}$, 2013; accepted December $24^{\text {th }}, 2013$

Copyright (C) 2014 Amjad Fayoumi et al. This is an open access article distributed under the Creative Commons Attribution License, which permits unrestricted use, distribution, and reproduction in any medium, provided the original work is properly cited. In accordance of the Creative Commons Attribution License all Copyrights (c) 2014 are reserved for SCIRP and the owner of the intellectual property Amjad Fayoumi et al. All Copyright (C) 2014 are guarded by law and by SCIRP as a guardian.

\section{ABSTRACT}

Nowadays, Model Driven Development (MDD) is a powerful technique for modelling complex systems and also for aligning business and information technology (IT), giving designers the ability to execute business models as they are intended to be run and behaved in the business environment. The Object Management Group (OMG) adopted many business standards into the Model Driven Architecture (MDA) environment e.g. Semantics of Business Vocabulary and Business Rules (SBVR), the Business Motivation Model (BMM), Business Process Modelling and Notation (BPMN) and Organisational Structure Model (OSM). These can work together to model different aspects of the enterprise at the business level. However, these models lack reasoning and dynamic simulation: there is no significant way to simulate discrete and continuous time system behaviour or to build arguments for reasoning about the design options within the OMG specifications. In this paper we propose an approach to overcoming this problem, by integrating a set of modelling tools into one single platform, taking advantage of meta-modelling techniques to integrate new tools with the OMG specifications in a model driven environment. The prospective framework should be able to tackle advanced analysis and design problems by intertwining design, reasoning and simulation to achieve a higher level of design maturity through implementing the desired scenario.

\section{KEYWORDS}

Enterprise Modelling; Model Driven Development; Business Modelling; Models Integration; Complex and Dynamic Enterprise Modelling Framework

\section{Introduction}

Enterprises are seen as socio-technical systems, and their analysis and design remain a continuous challenge. Baxter and Sommerville [1] argued that IT projects fail because they do not recognise the social and organisational complexity of the environment. Sterman [2] argued that it was important to expand mental and thinking boundaries, and to move from generalisations about accelerating learning and systems thinking to developing actual tools and processes that help us understand complexity, design better operating policies, and guide change in sys- tems in all scales. However, to solve such an issue, we need tools that help us recognise this complexity and support full alignment to move from understanding the whole environment to designing operations, policies and information systems [3]. Morabito [4] recommended that organisations should consider several stages of alignment, including dynamic alignment, in which it is fundamental for any modelling language to incorporate static and dynamic ontology in process dynamics [5]. To respond efficiently to such challenges and achieve these aims, the enterprise model (EM) should be dynamic and agile [6]. 
However, collaboration, agreement and faithfulness to the language used by stakeholders are important in order to facilitate analysis and share knowledge across a project [5].

It is not easy to decide what is required to handle issues such as complexity in either social or technical systems; likewise, a wide spectrum of analysis tools and mechanisms have been proposed, each with its own specialties and limitations. The MDD approach has been extended from software development to cover all aspects of an enterprise (social systems and technical systems) and this has served to enlarge the scope of the problems [7]. Still, MDD must own up to limitations in handling some types of problems in the social and business domain, and it is the aim of this paper to contribute to filling this gap.

The research questions, which this paper investigates and for which it attempts to offer insights, are as follows:

1. What essential knowledge needs to be covered in an enterprise modelling platform?

2. What analysis process, mechanisms and tools are required to provide comprehensive and efficient enterprise modelling?

3. How can we integrate these modelling tools into a single model driven platform?

The paper is organized as follows: Section 2 describes the motivation and main challenges facing current enterprises in the modern socio-technical environment. Section 3 provides a background to MDD and the wellknown MDA approaches. Section 4 contains a proposal of a hybrid approach that can address the current challenges. Section 5 draws conclusions and discusses future work.

\section{Complexity and Dynamicity Problems in an Enterprise Context}

The socio-technical environment is characterised by complexity, evolution and uncertainty. Evolution is a phenomenon describing the process of change which occurs in all ecological and non-ecological systems [8], while uncertainty results from the limitations of the human mind's capability and knowledge to describe or understand certain phenomena, especially those caused by hidden or unnoticeable relationships and dependencies between change influences (influencer and influenced). The environment and the physical universe we live in continuously evolves, and this is also true for all of the sub-entities of the universe, i.e. societies, ecology, technology, theory, science and cognitive stability: all evolve over time in their own contexts. For a long time, researchers have tried to analyse complex situations occurring in our world, including behaviours, unexpected statuses and many other social and ecological phenomena.
However, the best way to resolve the debate is by paying attention to multiple levels of analysis of systems, groups and individual interactions with a dynamic focus on how systems and sub-systems behave while communicating within their environment. While the contexts of business performance provide turbulent environments with high uncertainty and ambiguity, business value interactions in complex systems $[9,10]$ typically show characteristics such as multi-scale interactions with high contingency and nonlinearity, emergent behaviour, pattern formation and self-organisation.

Clearly, the new socio-economic and socio-technical systems operate with more efficiency, speed and accuracy, but at the same time they have increased in complexity and dynamicity. Thus, in an attempt to manage and control the new innovated systems on the one hand, and increase the number of influencing factors on the other, even the most optimistic ICT design initiatives have found the rhetorical moves unwieldy or struggled with the software tools to express and manage matters as they would like. Complex "nonlinear" situations entail unfamiliar flow and unexpected sequences and are mostly not visible or understandable. Sterman [2] clarified the importance of supporting decision-making by expanding the boundaries of mental models to understand complex possible behaviours and results: this will not be possible without intensive simulation of dynamic complex behaviours and reasoning about design choices.

It is understood that for successful enterprise modelling implementation we need competent people, a holistic framework "blueprint", effective processes and supportive tools. Model Driven Development tools are considered as advance tools to develop complex systems and these have been expanded in the last few years to cover a wider scope than the usual software application, such as complex systems engineering and enterprise business modelling.

\section{Background to MDD}

Model Driven Development (MDD) is a new paradigm for software development that focuses on the idea of model transformation and model artefacts mapping. The transformation between models is achieved in several ways, for instance: 1) Horizontal transformation: transferring between models has the same level of abstraction. 2) Vertical transformation: transformation between models with different levels of abstraction/details e.g. the transformation between business model and technical model and vice versa [11]. MDD has been improved and supported by the engineering approach, Model Driven Engineering (MDE). Initially, OMG developed the MDA approach so that the assets and IT resources of an organisation could be integrated and maintained [12]. MDA includes techniques that allow organisations to facilitate 
and make changes in their software architectures that could be represented in models to code and/or reversing code into models for legacy applications. MDA is a generic approach and does not define a specific development methodology.

The MDA consists of three layers of models: 1) Computation Independent Model (CIM), 2) Platform Independent Model (PIM), and 3) Platform Specific Model (PSM). The transformation among these layers has received much attention from the research community, mainly the transformation from PIM to PSM level. Nowadays the focus of model driven approaches has shifted from technical transformation from PIM to PSM, to business and enterprise aspects; these lie at the centre of the new specifications adopted by OMG, and more specifications are currently under consideration from OMG. These models at most fall under the business or software requirements area in the CIM level "in MDA layers" where the artefacts embedded in the business, environment and people need to be extracted and captured to develop IT systems $[11,12]$. These models will significantly improve the business-IT alignment and facilitate change. Chapin [13] named the CIM models the system environment's models in a new MDA proposal. The focus becomes how these models will be integrated and transferred to the PIM layer. The MDA generalisation proposal is a comprehensive model that could apply to any kind of system, i.e. if we consider the organisation environment as the widest limit of organisation enterprise models, and then the system application environment is a subset of the organisation environment.

This generalisation has allowed MDA to be applied as example to organisational models or any non-software/IT system [13]. In this case, the environment will be those features that fall outside the organisation related to the political, economical or ecological environment, legal, market, competitors and so on. To build such a model, first we need to identify the essential enterprise models, which are the activities that should be conducted by the organisation to deliver value in a very fundamental manner without any consideration of technology or supportive tools. The PIM and PSM levels consider the technology needed to support these activities. PIM includes independent software models that are not specific or related to any technology, while the PSM includes software models related to specific technology, e.g. Java, .NET, $\mathrm{C}++$ and $\mathrm{DB}$.

In this case, the PIM and PSM models are various in MDA applications to others and also we could have several PIM and PSM models for the single system to offer a full description of the system implementation. However, the point of view of the layers also could be vary, the PSM level in organization will also look as a PIM level in the IT system model, where we need to consider an- other PSM for farther implementation details.

Nevertheless, OMG adoption of BMM specification was the first stage of realization of the notion of MDA wide applicability to any type of systems, this offer a direction to business architecture modelling. OMG notion is to use a combination of models that allow organizations to have comprehensive and semantically aligned enterprise view using the fallowing specifications:

- BMM [14] to define business "End”, "Mean”, “Assessment" and "Influencers".

- SBVR [15] for terminology and ontology to insure strong applying and smooth transformation as the concepts, vocabulary and fact which will be the basis for building business rules and policies.

- OSM [16], for roles, responsibilities and organization structure (units, groups and individuals).

- And finally BPMN [17] to model business inter and intra processes.

These are to be followed by abstract and detailed ISs design fully aligned to enterprise models using OMG specification such as SysML, UML [18] and SoaML [19]. These specifications were fully or partly implemented in several enterprise modelling tools such as IBM Rational System Architect ${ }^{\circledR}$ and KnowEnterprise/ $/ \mathrm{IT}^{\circledR}$ software.

MDD includes more than just the models themselves. Meta-models, transformations between models, a process for creating and managing the models and their transformations, as well as tools to support the process, are all necessary for an MDD approach to serve its purpose [20]. MDD is also concerned with uncovering the implicit knowledge that modelers rely on when designing models [21]. This knowledge is encoded in clearly defined rules for transforming models. The existence of such rules would facilitate subsequent changes to the system, e.g. to fix errors or to extend the functionality.

\section{Hybrid Enterprise Modelling Approach}

\subsection{Essential Knowledge for Enterprise Modelling}

In order to analyse and design complex and dynamic systems efficiently, it is important to understand how to break down these systems to different sets of knowledge ontologies. Jurisica et al. [22] classified knowledge into four sets of ontology types; static ontology, dynamic ontology, intentional ontology and social ontology. Some of these ontologies, mainly the static and dynamic ontology, are well covered in the OMG specifications.

While in another approach, seven types of ontologies have been distinguished in an enterprise context. The focus will be on essential enterprise models represented by the seven S's ontologies [23]. The contextual approach for ontology [23] that distinguishes seven contextual domains; they are (purpose, actor, action, object, 
facility, location, and time), structuring the concepts within and between the domains is guided by the seven S's scheme:

Somebody (actor); does something (action); for some reasons (purpose); for someone (object); with the help of something (facility); somewhere (location) and sometimes (time).

These ontologies clearly can help in defining essential aspects that need to be covered within any enterprise modelling effort, which is that the enterprise aspects are essential in its essence and independent of whether people or automated equipment will do the work and very generic to any type of enterprise. These models are used as a starting point to make decisions about the design, independent of any technology and whether these technologies can be mainly used for designing any class of platform specific models "part of PSM". The essential enterprise models that models inter and intra business processes, organization, motivation, and rules are characterized by the following:

- Focuses on bringing about essential aspects to create value, directly or indirectly by communication and interaction between stakeholders.

- Including knowledge that is important to drive requirements of bottom level tasks for kinds of business facts as defined in the business ontology and vocabulary.

- How tasks are fulfilled and by whom, still hidden details excludes all (no information-processing considerations).

- Independent from information processing, transformation, document, data, storage or information artefacts and technology.

\subsection{Analysis Tools and Process for Enterprise Modelling}

In the area of Enterprise Modelling there is a vast number of modelling languages on offer to assist in capturing and conveying particular information, to support human understanding and reasoning on a certain topic. A typology of these modelling languages considers goal modelling c.f. [24], rule modeling [15,25], and business process modeling [17] being some of the prominent approaches. We have many tools based on OMG specifications to handling enterprise and represent it in a formal mode, as example business ontology, which can be represented using SBVR. This came from natural language ontology and policy resources, which should be offered business owner and regulations bodies, such as information should be integrated with essential enterprise models to establish the basis of required knowledge for communication and performing tasks.

The transformation is the result of essential enterprise models after applying data requirements of tasks to ontology presented as a manual information-processing component of executable tasks and rest of class of PIM and vendor's PSM in an enterprise models stage. However, the transformation can produce business requirements for system or software as consolidated data and rules requirements, which give business the means of recorded data that may contain additional functional, performance and design requirement.

In this activity, a responsibility for negotiation with IT supplier-side in information systems model is a part of the design process, rationality, factuality and causality of the design choices are very crucial. Exchanging information about enterprise requirements to satisfy these requirements by confirming the suitable design; in parallel, in software development side it will generate sequence of transformations in order to transfer enterprise models to IT data semantic represented as PIM with different levels to PSM in the end. Finally, the business customer and IT supplier parts are representing the knowledge of what is about the 'record data' and buckets that hold data. Critical analysis of people, processes and information including understanding the dynamic behaviour is essential during the enterprise and IT design and development. It is recommended to start the analysis with high level enterprise goals, "motivation", later to be aligned to operation and IT levels.

Current EM approaches are to a large extent able only to configure systems that are within one platform or within the same vendor alliance. This restricts the range of functionality that can be realized as well as limiting the deployment alternatives such as cloud services. In the latter case, a particular challenge is the need to consider the business model of the cloud service provider as well as compliance to various non-functional requirements, which in turn may require redesigning an organization's business model for the particular product or service.

The MDA is applicable to large-scale concurrent and distributed system that is comprised of complex systems. MDA considered as practical realization of "System of Systems" notion, by building systems models that are aligned, with strong semantic relations among them. All OMG specifications have what is called the underlying interoperability meta-model to allow models technical mapping and execution, the models transformation from business models to system design models then to code is responsibility of the tool vendor to provide such as complete tool for the business-modelling tool i.e. the BPMN tools that generate BEPEL or XBPDL files.

By using OMG specifications including UML models, it is possible to describe the essential ontology of any enterprise model. However, still testing the ontology behaviour and social reasoning about its designing is not covered by these specifications, therefore integrating 
these modelling tools with system dynamics modelling and design rationale become crucial. BMM will act as an enterprise profile that incubates the other OMG specifications, in the same way new tools to increase the analysis and design capability to cover social and dynamics ontology should be integrated. The proposed approach integrate three types of modelling mechanisms 1) Enterprise Modelling, 2) Design Rationale and 3) System Dynamics Modelling in one model driven platform, it is a top-down modelling approach to model enterprises, where every modelling stage contain reasoning about design rationale and simulation of business dynamics. The process flows from high level motivation (End and Mean) to operation and processes then to IS and IT. The operation management should continue monitoring and assessing operation results against pre-defined KPI's that linked to enterprise motivation.

MDD helps by creating mature information systems and by completely aligning the development effort of business and social requirements to software and system development in a systematic way. It can represent static and design-time dynamic ontology efficiently. MDD plays a core role in this research, focusing on MDD business modelling specifications proposed by OMG. Dynamic modelling that use system dynamics modelling [2] tools will help to simulate and forecast for business design, this to represent dynamic ontology in the runtime and simulate the behaviour to offer better insight before designing systems. Design Rationale [26] will offer qualitative rea- soning to improve decision making about design options, this technique will help to fulfill intentional ontology and level of social ontology, since this technique, offers the ability to collaborative thinking and brain storming between stakeholders. The following Figure 1 represents how the tools will cover each particular enterprise modelling aspect.

This strategy will cover the important aspects of enterprise modelling and use reasoning and dynamics to offer the enterprise insight and agility to fulfil dynamic artefacts. Exploring design space and dynamic simulation of future state will feed the enterprise modelling design, the correlations between people, systems, artefacts and contextual influencers should be understood. The impact of each on the entire enterprise can be examine qualitatively and quantitatively in iterative analysis and design way in order to cope with co-evolutionary nature of the socio-technical systems.

The goal is to provide a better understanding of complex systems to develop the enterprise driven architecture. The essential ontologies are satisfied by using the suggested modelling tools and described as in the following Table 1.

Here it is necessary to mention the need of the MDA approach to develop interoperability capable enterprise that can integrate and transform amongst different enterprise levels (strategic, tactical, operational and ISs) and different tools (enterprise modelling, design rationale and system dynamics modelling).

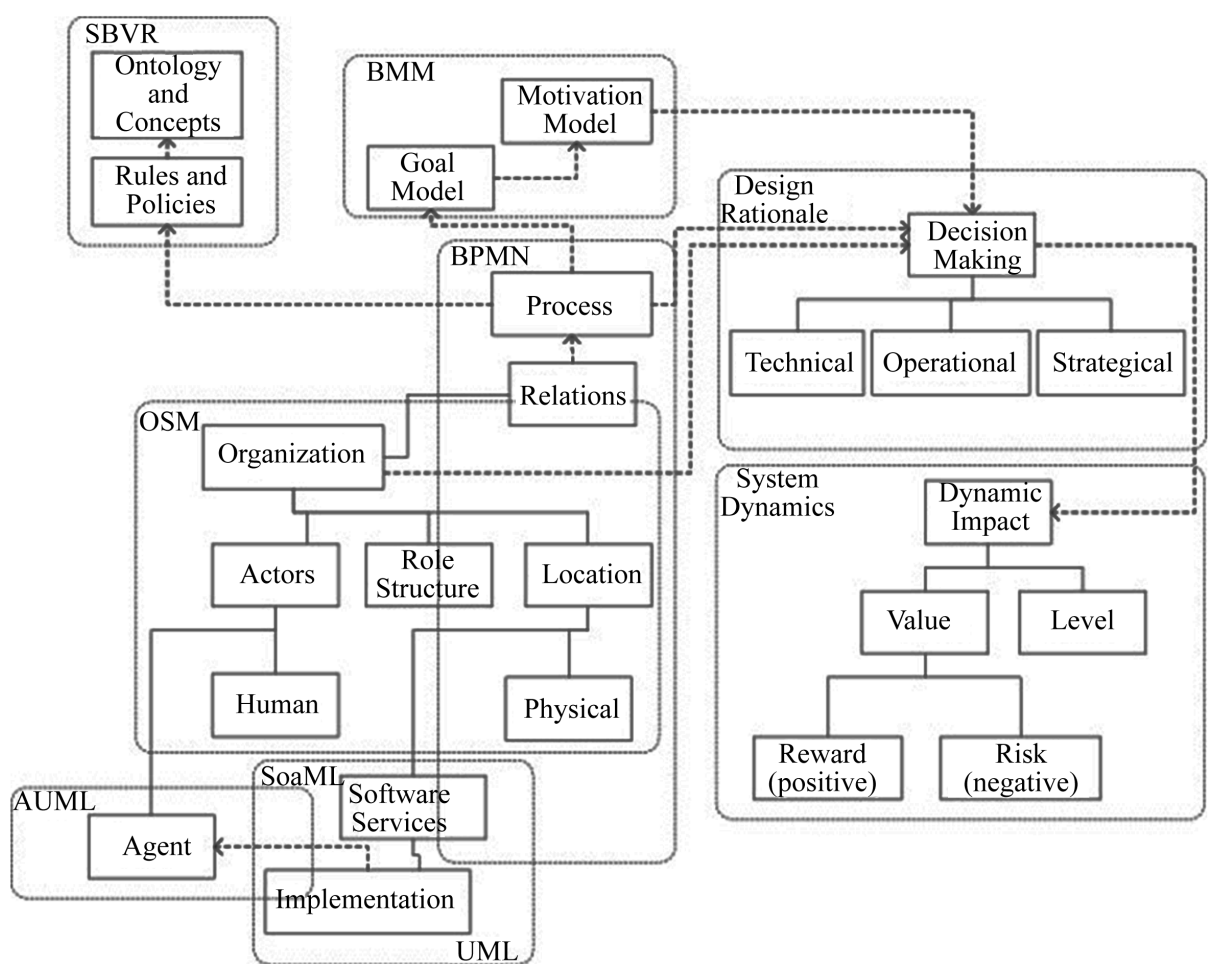

Figure 1. Enterprise modelling and supportive languages associated to each area of the enterprise modelling. 
Table 1. Enterprise essential ontology and modelling tools mapping.

\begin{tabular}{ll}
\hline $\begin{array}{l}\text { Essential Ontology (Seven S's + } \\
\text { Dynamic and Social) }\end{array}$ & OMG Specifications \\
\hline Somebody (actor) & OSM and BPMN \\
Do Something (action) & BPMN and OSM \\
For Some Reasons (purpose) & BMM \\
For Someone (object) & BPMN \\
With the Help of Something (facility) & OSM, SBVR and UML \\
Somewhere (location) & BPMN \\
Sometimes (time) & BPMN and SBVR \\
Social Judgment and Reasoning & Design Rationale \\
Dynamic Simulation & System Dynamics Modelling \\
\hline
\end{tabular}

The new framework also will response to OMG declaration about its vision to integrate dynamic model with their MDA environment, considering dynamic models in MDA bring two important benefits:

- Moving from dynamic model as a simulation technique, which offers an insight about future states, options and decision also implementation capable using one of the OMG implementation standards. Thus, to enforce policies/rules of specific desired status on implementation models.

- Provide a dynamic technical framework reflecting the real change in the environment rather than the old static infrastructure, by establishing set of structured rules and event based rules to response to potential events "increase the agility and fast adaptation."

Figure 2 shows how the developed approach can be used for modelling and developing enterprises in the methodological (top-down) goal orientated process.

\subsection{Implementation in a Model Driven Platform}

The embedded capabilities of the approach help to understand and plan for various types of socio-technical change. The developed framework is based on integrating coherent set of models of enterprise modelling, design rationale and system dynamics to offer analysis, simulation and implementation capabilities for activities engaged in the enterprises. This hybrid-modelling approach offers capabilities of reasoning, simulation and IT-business alignment while considering both social and technical aspects for analysing and designing enterprises. The tools proposed (BMM, BPMN, SBVR, OSM, System dynamics and Design rationale) for enterprise activities and (UML, SysML, SoaML and AgentUML) for software design and implementation. These all helped to collect enterprise knowledge, understand the enterprise and its environment, and align social, business and technical aspects in systematic manner.

The realization of the integrated models lay in achiev- ing semantic interoperability, the first step is to build metamodels for design rationale and system dynamics models. Using ontology development, it is possible to match the concepts between different metamodels [27]; every model should have a metamodel and ontology model to enable the semantic interoperability between metamodel concepts. Later it will be possible to transfer these models to implementation models in PIM and then PSM models, as described in Figure 3.

The implementation specification of the integrated approach based on eclipse platform to create meatmodel as UML profiles to store the data models, mainly we will use Eclipse modelling tools and Eclipse ATL for Ontology Definition Metamodel (ODM) implementation, ODM defines five metamodels (RDFS, OWL, Topic Maps, Common Logic and Description Logic), two UML Profiles (RDFS/OWL Profile, Topic Maps Profile) and a set of QVT mappings from UML to OWL, Topic Maps to OWL and RDFS/OWL to Common Logic. This will help to build adapters for system dynamics and design rationale tools by transferring the data file to common file standard based on ontology specification (OWL and RDF) to insure interoperability. This can be beneficial in defining domain knowledge and selecting the suitable scenario that needs to be executed through specific tool in the integrated approach.

\section{Conclusion}

Due to the rapidly changing business environment and increasing of its complexity, the formal abstraction and conceptual modelling have some limitations in providing reasoning and dynamic simulation; taking advantage of other modelling and simulation mechanisms becomes crucial. Toward this end, this paper presents a novel model driven approach integrating a set of enterprise modelling techniques to dynamics and reasoning modelling techniques by integrating their tools into the model driven development platform, using metamodelling and ontology for semantic interoperability. This effort considers what has been missing within the model driven development environment. The integration with system dynamics tools will bring simulation capability into MDD environment, likewise, with the integration with design rationale tools to bring reasoning and argumentation to MDD environment. The hybrid approach has been tested on several business case studies, and this position paper aims to describe prospective work to be carried out in building a metamodel for dynamic and reasoning modelling to integrate them to a set of enterprise modelling specifications proposed by OMG in one platform toward "one-click" transformation among different analytical, design and implementation tools. Future work, respectively, will consider providing examples of how these models will work 


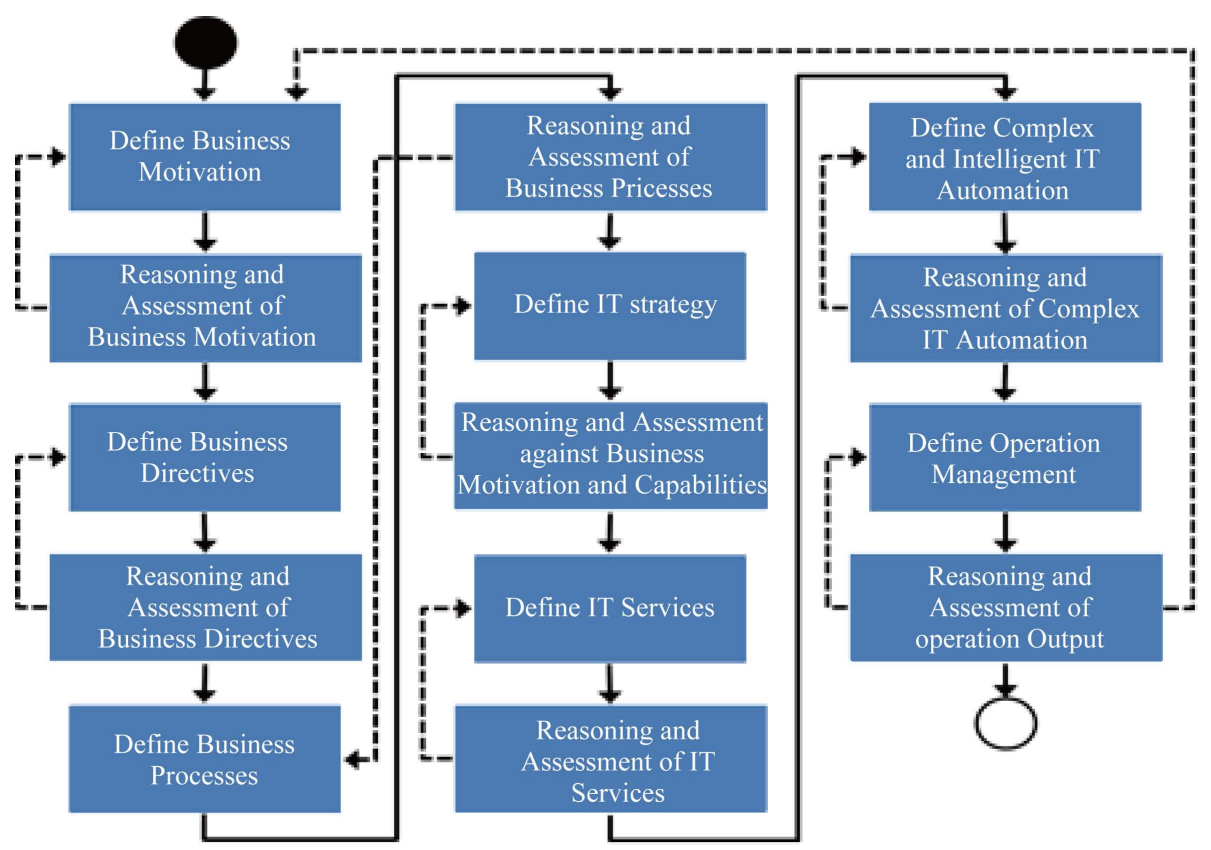

Figure 2. The proposed development process for top-down analysis and design.

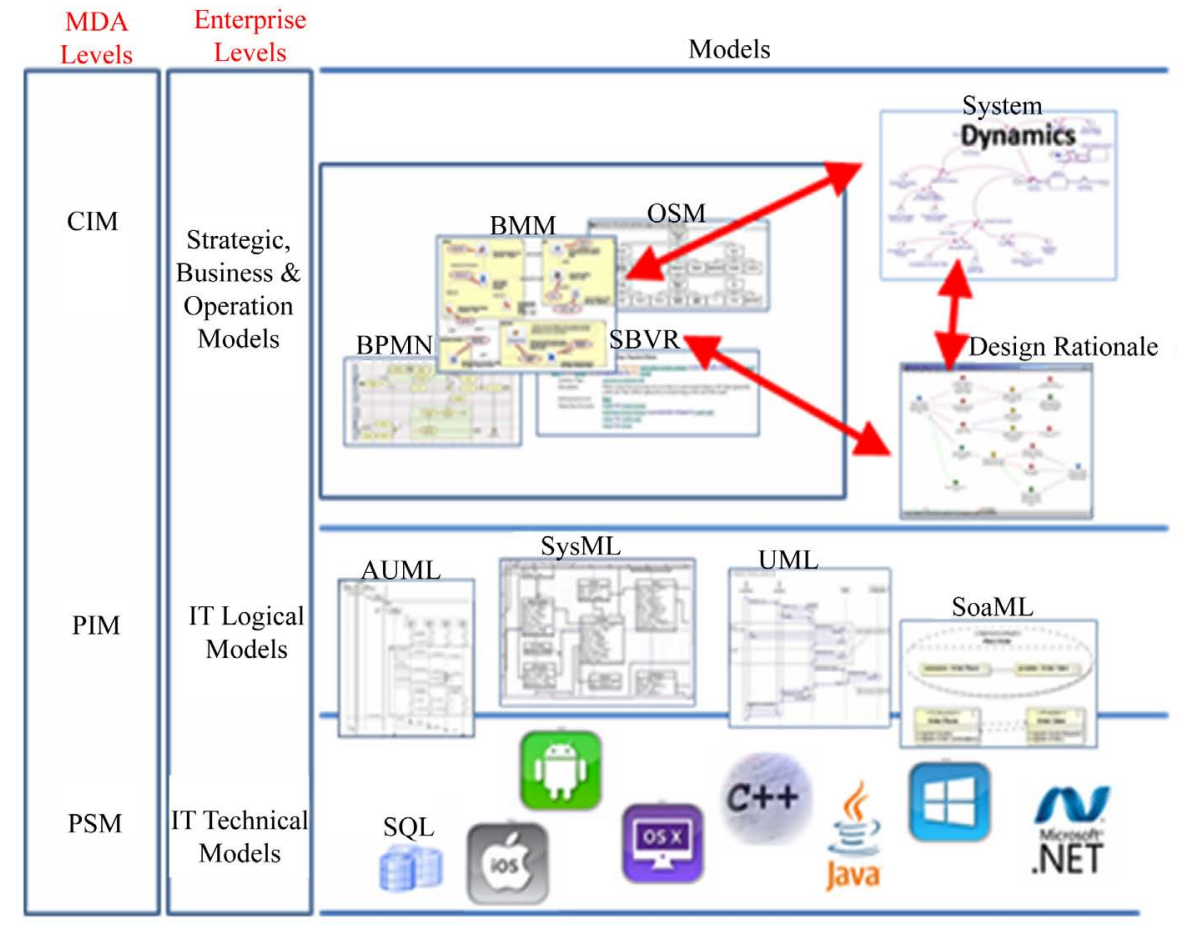

Figure 3. The hybrid enterprise modelling approach and MDA.

together, build metamodels for dynamics and reasoning modelling, and integrate these tools with the development environment platform using ontology to exchange files/data among these tools.

\section{Acknowledgements}

We would like to thank the anonymous reviewers for their valuable constructive criticisms and feedback on an early version of this paper, which helped to improve the argument, structure and presentation of the work.

\section{REFERENCES}

[1] G. Baxter and I. Sommerville, "Socio-Technical Systems: From Design Methods to Systems Engineering. Interact- 
ing with Computers,” 2011. http://dx.doi.org/10.1016/j.intcom.2010.07.003

[2] J. D. Sterman, "Business Dynamics: Systems Thinking and Modeling for a Complex World,” Irwin/McGraw-Hill, Boston, 2000.

[3] P. Loucopoulos, “The F3 (From Fuzzy to Formal) View on Requirements Engineering,” Ingénierie des Systèmes d'Information, Vol. 2, No. 6, 1995, pp. 639-655.

[4] J. Morabito, I. Sack and A. Bhate, "Organization Modeling: Innovative Architectures for the 21st Century,” Prentice Hall PTR, Upper Saddle River, 1999.

[5] E. S. Yu, "Social Modelling and i*. Conceptual Modeling: Foundations and Applications," 2009.

[6] N. R. Terkelsen, “Architect in the Spotlight," Interview in Journal of Enterprise Architecture, Vol. 9, No. 1, 2013, pp. 2166-6792.

[7] P. Loucopoulos, "From Information Modelling to Enterprise Modelling. Information Systems Engineering: State of the Art and Research Themes,” In: S. Brinkkemper, E. Lindencrona and A. Solvberg, Eds., Springer, 2000, pp. 67-78.

[8] P. J. Bowler, "Evolution: The History of an Idea," 3rd Edition, University of California Press, 2003.

[9] N. Nohria and R. Gulati, "What Is the Optimum Amount of Organizational Slack? A Study of the Relationship between Slack and Innovation in Multinational Firms," European Management Journal, Vol. 15, No. 6, 1997, pp. 603-611.

[10] D. Tapscott, "Creating Value in the Network Economy," Harvard Business School Press, Boston, 1999.

[11] O. Pastor, S. España, J. I. Panach and N. Aquino, "ModelDriven Development," Informatik-Spektrum, Vol. 31, No. 5, 2008, pp. 394-407. http://dx.doi.org/10.1007/s00287-008-0275-8

[12] OMG, “MDA Guide Version 1.0.1,” 2003. http://www.omg.org/mda

[13] D. Chapin, "Business Architecture as the Application of the MDA Foundation Model to 'Organizations'," Presentation to Open Group Business Architecture Working Group, Glasgow, 2008.
[14] OMG, “Business Motivation Model (BMM) 1.1,” 2010.

[15] OMG, "Semantic of Business Vocabulary and Business Rules (SBVR) 1.0,” 2008.

[16] OMG, “Organization Structure Metamodel (OSM) 2009. Revised Submission, bmi/09-08-02,” 2009.

[17] OMG, "Business Process Model and Notation (BPMN) 2.0,” 2011.

[18] OMG, "Unified Modeling Language (UML), Superstructure, V2.1.2,” 2007.

[19] OMG, "Service Oriented Architecture Modeling Language (SoaML), Version 1.0.1,” 2012.

[20] S. Kent, "Model Driven Engineering. Integrated Formal Methods”, Springer, 2002.

[21] P. Louridas and P. Loucopoulos, "A Generic Model for Reflective Design,” ACM Transactions on Software Engineering and Methodology, Vol. 9, No. 2, 2000, pp. 199237. http://dx.doi.org/10.1145/350887.350895

[22] I. Jurisica, J. Mylopoulos and E. Yu, "Ontologies for Knowledge Management: An Information Systems,” Knowledge and Information Systems, Vol. 6, 2004, pp. 380401.

[23] M. Leppänen, “A Context-Based Enterprise Ontology. In Business Information Systems,” Springer, Berlin/Heidelberg, 2007, pp. 273-286.

[24] E. Kavakli and P. Loucopoulos, "Experiences with GoalOriented Modelling of Organisational Change," IEEE Transactions on Systems, Man and Cybernetics-Part C, Vol. 36, No. 2, 2006, pp. 221-235. http://dx.doi.org/10.1109/TSMCC.2004.840066

[25] A. Fayoumi and L. Yang, "SBVR: Knowledge Definition, Vocabulary Management, and Rules Integrations,” International Journal of E-Business Development, 2012.

[26] W. C. Regli, et al., “A Survey of Design Rationale Systems,” Springer-Verlag, 2000, pp. 209-235.

[27] D. Karagiannis, H. G. Fill, P. Hofferer and M. Nemetz, "Metamodeleing: Some Application Areas in Information Systems. In Information Systems and e-Business Technologies,” Springer, Berlin/Heidelberg, 2008, pp. 175-188. 